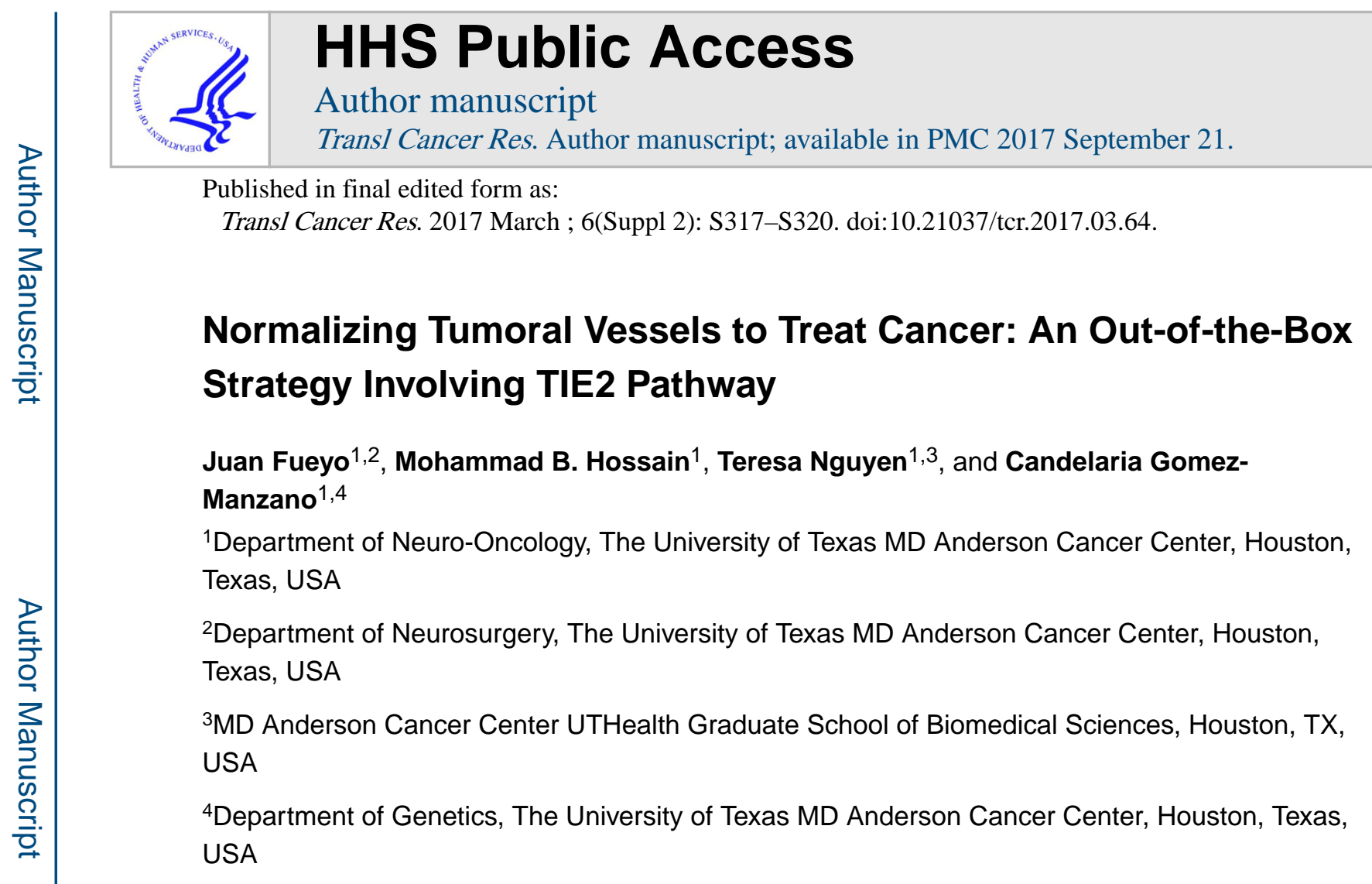

\title{
Angiogenesis and Cancer
}

The idea of targeting vessels for cancer therapy, termed antiangiogenesis, was coined by Folkman in his pioneer report in $1971(1,2)$. Folkman's seminal contribution to cancer biology was the early description of what we now understand as the tumor microenvironment (2). In this manuscript, he postulated that tumor growth depends on vessel recruitment and that the growth of vascular and tumor cells are interdependent. Per instance, he stated that the maintenance of the mitotic index of the two cell populations depends on each other, and that the secretion of diffusible factors from tumor cells influences the formation of tumor capillaries (1). Furthermore, he described that the blockade of proangiogenic signals resulted in the regression of the new blood vessels. In addition, he hypothesized that central tumor necrosis is the consequence of poor perfusion due to increased internal pressure and decreased of the blood flow at the tumor site $(1,2)$. This hypothesis was tested by Rakesh Jain who demonstrated that Folkman's assumption was correct, and further suggested that increased interstitial fluid pressure might impede the delivery of large anticancer agents to tumors $(2,3)$.

\section{Antiangiogenic Therapy}

The conceptual basis of antiangiogenic therapy was based on eradicating tumors by destroying their vascular structures and, subsequently, depriving cancer cells of nutrients and oxygen. Although the description of a blood vessel growth stimulator factor was dated in 1939 (4), the discovery of vascular endothelial growth factor, VEGF, was what constituted

Correspondence to: Candelaria Gomez-Manzano, MD (cmanzano@mdanderson.org).

Conflicts of interests.The authors declare no conflict of interest. 
the first tangible milestone toward an antiangiogenic therapy $(5,6)$. Years of exciting research in this area culminated with the development of bevacizumab, a recombinant humanized VEGFA-specific monoclonal antibody. Bevacizumab was subsequent approved by the FDA in 2004 as the first-line treatment for metastatic colorectal cancer (7), and later approved in 2009 for a second line treatment for glioblastoma (8). These advancements inspired the development of many other VEGFA signaling pathway inhibitors such as sunitinib, sorafenib, aflibercept, and ramucirumab (4). Despite the initial excitement and some modest benefits observed in clinical trials, the administration of antiangiogenic therapy as single agents did not yield long-term survival benefits. For instance, treatment with bevacizumab did not improve survival in two major studies of patients with glioblastoma (8). For that reason, ongoing studies are in place to further understand the molecular mechanisms of resistance of solid tumors to anti-VEGF therapy.

\section{Normalization of Tumoral Vessels}

While angiogenesis is a hallmark of tumors, tumor vascular structures are architecturally and functionally abnormal. These vessels are tortuous with increased permeability that results in higher interstitial fluid pressure, further challenging the arrival of oxygen and nutrients. Despite this fact, it is worthy to note that antiangiogenic therapy administered in combination with chemotherapy in patients with metastatic colon cancer produced an unprecedented increase in survival (7). Multiple mechanisms might be behind this effect, such as the combinatorial therapy being able to target multiple compartments within the tumor including endothelial and cancer cells (9). However, Jain's group has extensively worked to test an alternative, and perhaps a more relevant, hypothesis. According to this group of investigators, by applying antiangiogenic agents to the tumor, the vasculature will be normalized to allow proficient delivery of oxygen and drugs to the cancer cells $(10,11)$. In agreement with this model, VEGFR2 inhibition transiently normalized glioma vasculature resulting in a reduced intracranial edema and improved response to radiotherapy of mice bearing gliomas $(12,13)$. Further supporting Jain's hypothesis, results from phase II trials showed an association between parameters related to vascular normalization, such as increased perfusion and oxygenation, with survival. Thus, treatment with cediranib induced transient vessel normalization in patients with recurrent gliomas, measured as decreased microvesssel diameter and permeability, effectively reducing edema $(11,14,15)$. The vessel normalization effect was not restricted to brain tumors and was also observed in other kind of cancers. Per instance, tumors from patients with HER2-negative breast cancer treated with bevacizumab showed signs of vascular normalization, such as increased pericytecovered microvascular density and decreased interstitial fluid pressure (16).

\section{TIE2 Pathway as an Alternative Target Approach to Normalize Tumoral Structures}

The time window of opportunity to utilize (i.e. administer additional therapies) the vessel normalization phase after anti-VEGF/VEGFR2 is too narrow and therefore other strategies are being explored. These approaches are mainly focused on targeting the TIE2/ Angiopoietin pathway. TIE2 is a tyrosine kinase receptor initially described in the 
membrane of endothelial cells and hematopoietic precursor (17), that has later been found to be expressed in other non-endothelial cells, such as cancer cells, pericyte precursors, and specific monocytic populations (18-20). TIE2 signaling is regulated upon binding to its ligands, angiopoietins, such as Angiopoietin1 (ANG1) and 2 (ANG2) which play a dynamic role in vessel formation, maintenance, and permeability. Thus, ANG1 stimulation of TIE2 tightens endothelial junctions resulting in blood vessel stabilization. ANG2 is often upregulated in gliomas, and plays a role in vessel destabilization in low VEGF levels conditions, or a pro-angiogenic role in high VEGF levels conditions (20). Interestingly, ANG2 has been reported to be overexpressed in tumors after anti-angiogenic therapies (14, 21 ), and it is known to act as a chemoattractant of TIE2-expressing monocytes (21). Overrepresentation of this specific monocytic population might be related to the tumor escape to antiangigogenic therapies (18). In addition, ANG1/ANG2 ratio correlated positively with vascular normalization (14).

Several reports have previously tested the possibility of normalizing tumor vasculature to treat cancer by targeting both the VEGF and the TIE2/ANG pathway. In this regard, the dual inhibition of VEGF by cediranib and ANG2 by a neutralizing antibody, MEDI3617, proved to prolong the survival of mice bearing malignant gliomas (22). Recently, Park and colleagues (23) explored the effect of an antibody, ABTAA, which exerts a dual action on TIE2 pathway (ANG2-binding and TIE2 activating antibody) on three murine models of cancer: orthotopic implantation of gliomas, Lewis lung carcinoma, and spontaneous mammary cancer. The authors reported that TIE2 activation induced vascular normalization in the tumors, which resulted in enhanced blood perfusion. Of interest, chemotherapeutic drug delivery was increased and resulted in reduced tumor growth and metastasis. While an ANG2 blocking antibody (ABA) has some effect on these models, it was more moderate than the effect achieved using the (TIE2-ANG2) dual targeting. Thus, administration of ABTAA or ABA $(10 \mathrm{mg} / \mathrm{kg})$ to glioma bearing mice resulted to a $39 \%$ or $17 \%$ reduction of tumor volume, respectively. Park and colleagues suggest that the superiority of ABTAA versus $\mathrm{ABA}$ is due to the inability of ANG2 blocking agents to decrease tumor hypoxia, which significantly contributes to the increase in the levels of angiogenic factors and the invasive pattern of tumors observed after antiangiogenic therapies $(21,24)$. Interestingly, when ABTAA was combined with temozolamide, the standard chemotherapy for glioma treatment, the tumor volume was inhibited 75\%, suggesting that ABTAA enhanced drug delivery. In lung and breast cancer models, the effect of the new agent was also abscopal, decreasing metastasis formation (23). Another interesting aspect of this work lies in the changes observed on the immune cell infiltration. Park and colleagues also described in their manuscript that the treatment with ABTAA promotes the tumor infiltration and polarization of M1-like tumor associate monocytes due to the reduction of ANG2 and lactate, suggesting the production of a favorable anti-tumor microenvironment (23). This is of significance because escape and resistance to antiangiogenic therapy has been linked by several studies to the infiltration of tumor-associated macrophages $(18,25)$.

Interestingly, these authors previously reported the use of ABTAA in treating sepsis (26), a disease, like cancer, associated to inflammation, vascular leakage, and increased ANG2 levels $(27,28)$. Their data, using a novel model of murine severe sepsis (high-grade cecal ligation and puncture, CLP), showed that treatment of these animals with $10 \mathrm{mg} / \mathrm{kg}$ of 
ABTAA at 6 and 18 hours after CLP reduced cytokine storms and vascular leakage, and strengthened the endothelial glycocalyx. The antibody was also tested in other models of sepsis, such as intraperitoneal $S$. aureus inoculation or LPS injection. The use of ABTAA reduced significantly organ damage and sepsis mortality in the tested models (26). In summary, targeting the TIE2/ANG axis results in vascular normalization and reduces inflammation in sepsis.

We have recently reported the nuclear translocation of TIE2 upon ANG1 binding. Nuclear TIE2 seems to act as a resistance factor to genotoxic stress by modifying the epigenetic pattern of tumor cells (29). In this regard, nuclear localization of TIE2 is related to enhanced DNA repair and radioresistance (29). Further investigation is needed to understand if nuclear TIE2 is a key player also in the resistance to DNA-damaging chemotherapeutic agents, and if this function will be jeopardized by the administration of ABTAA or similar TIE2targeting agents.

\section{Conclusions}

For several decades, researchers have searched for drugs that will decrease the blood flow of tumors with the goal of depriving tumoral cells from oxygen and nutrients. The development of new biological agents that might normalize tumoral vasculature to improve the delivery of chemotherapeutic drugs into the core of tumors is a more recent strategy that needs to be further tested in the clinical settings. The possibility of reducing metastasis while 'helping' the tumor to obtain oxygen and nutrients seems counterintuitive but merits further investigation. Maybe, as Mina Bissell's theory of "dynamic reciprocity" states, in a healthy micro-environment tumor cells with abnormal genomes should be capable of becoming normal (30), and thus, reverting 'tumoral' vessels to 'normal' vessels to target the core of the tumor should be right way of proceeding. To achieve these objectives efficient manipulation of the TIE2/ANG pathway seems to be critical.

\section{Acknowledgments}

This work was supported by the National Institutes of Health (NIH) grants R01 NS069964 and P50 CA127001.

\section{References}

1. Folkman J. Tumor angiogenesis: therapeutic implications. N Engl J Med. 1971; 285(21):1182-6. [PubMed: 4938153]

2. Zetter BR. The scientific contributions of M. Judah Folkman to cancer research. Nat Rev Cancer. 2008; 8(8):647-54. [PubMed: 18633354]

3. Boucher Y, Leunig M, Jain RK. Tumor angiogenesis and interstitial hypertension. Cancer Res. 1996; 56(18):4264-6. [PubMed: 8797602]

4. Ferrara N, Adamis AP. Ten years of anti-vascular endothelial growth factor therapy. Nat Rev Drug Discov. 2016; 15(6):385-403. [PubMed: 26775688]

5. Keck PJ, Hauser SD, Krivi G, Sanzo K, Warren T, Feder J, et al. Vascular permeability factor, an endothelial cell mitogen related to PDGF. Science. 1989; 246(4935):1309-12. [PubMed: 2479987]

6. Leung DW, Cachianes G, Kuang WJ, Goeddel DV, Ferrara N. Vascular endothelial growth factor is a secreted angiogenic mitogen. Science. 1989; 246(4935):1306-9. [PubMed: 2479986] 
7. Hurwitz H, Fehrenbacher L, Novotny W, Cartwright T, Hainsworth J, Heim W, et al. Bevacizumab plus irinotecan, fluorouracil, and leucovorin for metastatic colorectal cancer. N Engl J Med. 2004; 350(23):2335-42. [PubMed: 15175435]

8. Fine HA. Bevacizumab in glioblastoma--still much to learn. N Engl J Med. 2014; 370(8):764-5. [PubMed: 24552324]

9. Teicher BA. A systems approach to cancer therapy. Cancer Metastasis Rev. 1996; 15(2):247-72. [PubMed: 8842498]

10. Jain RK. Normalizing tumor vasculature with anti-angiogenic therapy: a new paradigm for combination therapy. Nat Med. 2001; 7(9):987-9. [PubMed: 11533692]

11. Jain RK. Normalization of tumor vasculature: an emerging concept in antiangiogenic therapy. Science. 2005; 307(5706):58-62. [PubMed: 15637262]

12. Kamoun WS, Ley CD, Farrar CT, Duyverman AM, Lahdenranta J, Lacorre DA, et al. Edema control by cediranib, a vascular endothelial growth factor receptor-targeted kinase inhibitor, prolongs survival despite persistent brain tumor growth in mice. J Clin Oncol. 2009; 27(15):254252. [PubMed: 19332720]

13. Winkler F, Kozin SV, Tong RT, Chae SS, Booth MF, Garkavtsev I, et al. Kinetics of vascular normalization by VEGFR2 blockade governs brain tumor response to radiation: role of oxygenation, angiopoietin-1, and matrix metalloproteinases. Cancer Cell. 2004; 6(6):553-63. [PubMed: 15607960]

14. Batchelor TT, Duda DG, di Tomaso E, Ancukiewicz M, Plotkin SR, Gerstner E, et al. Phase II study of cediranib, an oral pan-vascular endothelial growth factor receptor tyrosine kinase inhibitor, in patients with recurrent glioblastoma. J Clin Oncol. 2010; 28(17):2817-23. [PubMed: 20458050]

15. Batchelor TT, Sorensen AG, di Tomaso E, Zhang WT, Duda DG, Cohen KS, et al. AZD2171, a pan-VEGF receptor tyrosine kinase inhibitor, normalizes tumor vasculature and alleviates edema in glioblastoma patients. Cancer Cell. 2007; 11(1):83-95. [PubMed: 17222792]

16. Tolaney SM, Boucher Y, Duda DG, Martin JD, Seano G, Ancukiewicz M, et al. Role of vascular density and normalization in response to neoadjuvant bevacizumab and chemotherapy in breast cancer patients. Proc Natl Acad Sci U S A. 2015; 112(46):14325-30. [PubMed: 26578779]

17. Dumont DJ, Yamaguchi TP, Conlon RA, Rossant J, Breitman ML. tek, a novel tyrosine kinase gene located on mouse chromosome 4, is expressed in endothelial cells and their presumptive precursors. Oncogene. 1992; 7(8):1471-80. [PubMed: 1630810]

18. Gabrusiewicz K, Liu D, Cortes-Santiago N, Hossain MB, Conrad CA, Aldape KD, et al. Antivascular endothelial growth factor therapy-induced glioma invasion is associated with accumulation of Tie2-expressing monocytes. Oncotarget. 2014; 5(8):2208-20. [PubMed: 24809734]

19. Lee OH, Xu J, Fueyo J, Fuller GN, Aldape KD, Alonso MM, et al. Expression of the receptor tyrosine kinase Tie2 in neoplastic glial cells is associated with integrin beta1-dependent adhesion to the extracellular matrix. Mol Cancer Res. 2006; 4(12):915-26. [PubMed: 17189382]

20. Martin V, Liu D, Fueyo J, Gomez-Manzano C. Tie2: a journey from normal angiogenesis to cancer and beyond. Histol Histopathol. 2008; 23(6):773-80. [PubMed: 18366015]

21. Cortes-Santiago N, Hossain MB, Gabrusiewicz K, Fan X, Gumin J, Marini FC, et al. Soluble Tie2 overrides the heightened invasion induced by anti-angiogenesis therapies in gliomas. Oncotarget. 2016; 7(13):16146-57. [PubMed: 26910374]

22. Peterson TE, Kirkpatrick ND, Huang Y, Farrar CT, Marijt KA, Kloepper J, et al. Dual inhibition of Ang-2 and VEGF receptors normalizes tumor vasculature and prolongs survival in glioblastoma by altering macrophages. Proc Natl Acad Sci U S A. 2016; 113(16):4470-5. [PubMed: 27044097]

23. Park JS, Kim IK, Han S, Park I, Kim C, Bae J, et al. Normalization of Tumor Vessels by Tie2 Activation and Ang2 Inhibition Enhances Drug Delivery and Produces a Favorable Tumor Microenvironment. Cancer Cell. 2016; 30(6):953-67. [PubMed: 27960088]

24. Mazzieri R, Pucci F, Moi D, Zonari E, Ranghetti A, Berti A, et al. Targeting the ANG2/TIE2 axis inhibits tumor growth and metastasis by impairing angiogenesis and disabling rebounds of proangiogenic myeloid cells. Cancer Cell. 2011; 19(4):512-26. [PubMed: 21481792] 
25. Shojaei F, Wu X, Malik AK, Zhong C, Baldwin ME, Schanz S, et al. Tumor refractoriness to antiVEGF treatment is mediated by CD11b+Gr1+ myeloid cells. Nat Biotechnol. 2007; 25(8):911-20. [PubMed: 17664940]

26. Han S, Lee SJ, Kim KE, Lee HS, Oh N, Park I, et al. Amelioration of sepsis by TIE2 activationinduced vascular protection. Sci Transl Med. 2016; 8(335):335ra55.

27. Parikh SM, Mammoto T, Schultz A, Yuan HT, Christiani D, Karumanchi SA, et al. Excess circulating angiopoietin-2 may contribute to pulmonary vascular leak in sepsis in humans. PLoS Med. 2006; 3(3):e46. [PubMed: 16417407]

28. Siner JM, Bhandari V, Engle KM, Elias JA, Siegel MD. Elevated serum angiopoietin 2 levels are associated with increased mortality in sepsis. Shock. 2009; 31(4):348-53. [PubMed: 18791490]

29. Hossain MB, Shifat R, Johnson DG, Bedford MT, Gabrusiewicz KR, Cortes-Santiago N, et al. TIE2-mediated tyrosine phosphorylation of $\mathrm{H} 4$ regulates DNA damage response by recruiting ABL1. Sci Adv. 2016; 2(4):e1501290. [PubMed: 27757426]

30. Bissell MJ, Hall HG, Parry G. How does the extracellular matrix direct gene expression? J Theor Biol. 1982; 99(1):31-68. [PubMed: 6892044] 